\title{
Weitere Untersuchungen über metastatische Dermatosen bei akuten bakteriellen Allgemeinerkrankungen.
}

\author{
Von \\ Eugen Fraenkel.
}

(Aus dem Pathologischen Institut der Hamburgischen. Universität.)

Mit 8 Textabbildungen.

Das Studium der sich bei akuten bakteriellen Allgemeinerkrankungen an der Hautdecke abspielenden Prozesse durch histologische Untersuchung von vital herausgeschnittenen, erkrankten Hautstückchen hat unsere Kenntnisse über das Wesen dieser, kurz als metastatische Dermatosen zu bezeichnenden, Affektionen wesentlich gefördert. Abgesehen von klaren anatomischen Vorstellungen über die Art der ihnen zugrunde liegenden Gewebsschädigung haben wir erfahren, daß der Kreis der bei manchen dieser Allgemeinerkrankungen als toxisch aufgefaßten Exantheme ein immer engerer geworden ist, daß es sich vielmehr fast a usnahmslos um eine direkte Invasion der das Allgemeinleiden verursachenden $\mathrm{Kran}$ kheitserreger in das $\mathrm{Ha}$ u tge we be handelt. Freilich, von einem völligen Abschluß unseres Wissens auf diesem interessanten Gebiet sind wir noch weit entfernt. Es hängt das zum Teil damit zusammen, daß bei manchen Allgemeinerkrankungen die Hautdecke sehr viel häufiger den Ansiedlungsort für die Erreger bildet als bei anderen, wie namentlich bei den durch Staphylokokken verursachten septico-pyämischen Zuständen, bei denen man von einer gewissen Polymorphie der Exanthemformen sprechen kann. Im Gegensatz dazu sind die a uf Streptokokkenwirkung zurückzuführenden metastatischen Ausschlagsarten sehr viel weniger häufig als jene und sind nur ausnahmsweise mit eitrigen Prozessen vergesellschaftet. Wir haben ferner durch Vergleich der durch den sog. Friedländerbacillus herbeigeführten und jener eigenartigen, als Ekthyma gangränosum bekannten, auf Einwirkung des Bac. pyoc. zu setzenden Hauterkrankungen mit den bei Blut-Staphylo- und -Streptomykosen auftretenden uns für berechtigt gehalten, den Satz aufzustellen, daß bis zu einem gewissen Grade eine nichtzu verkennende Abhängigkeit der metastatischen Hautveränderungen vou der Natur des Krankheitserregers besteht. Das gilt vor allem für die an den Hautefflorescenzen zu 
E. Fraenkel: Weitere Untersuchungen über metastatische Dermatosen usw. 387

erhebenden mikroskopischen Befunde. Als in dieser Beziehung lehrreichstes Beispiel dürfte die Fleckfieberroseole auf der einen und die Ekthymaefflorescenz a uf der anderen Seite anzuführen sein. Übereinstimmung zwischen beiden besteht insofern, als sowohl der, für das Fleckfiek er allerdings seiner Natur nach keineswegs sichergestellte Erreger, ebenso wie der das Ekthyma gangränosum hervorrufende Bac. pyoc. an den Gefäßen angreift. Aber die Art der Schädigung ist bei beiden eine ganz verschiedene.

Neben diesen, für einzelne Infektionskrankheiten besonders mikroskopisch sich als spezifisch erweisenden Veränderungen an der Haut beobachten wir bei anderen nur, den Eindruck von kleinen und kleinsten Extravasaten erweckende Herde, die namentlich bei manchen Fällen epidemischer Genickstarre in so großer Zahl auftreten können, daß der ganze Körper mit ihnen übersät erscheint. Erst in den letzten 4 Jahren hat diese Art der petechialen Fxantheme bei der Genickstarre das Interesse der Kliniker und Pathologen in hohem Maße erweckt, und es liegt von anatomischer Seite eine Anzahl einschlägiger Untersuchungen vor, die ein allerdings auch jetzt noch nicht völlig abschließendes Urteil über die sich dabei in der Haut abspielenden Vorgänge zulassen. Als Fazit der bisher vorliegenden Vorstellungen hat sich ergeben, daß die makroskopisch bald als kleinere, bald als gröBere Blutaustritte imponierenden Herde in der Haut histologisch einen ganz verschiedenen Bau aufweisen können, daß also die Befunde dabei keine einheitlichen sind. Das haben die Untersuchungen von Benda (Berl. klin. Wochenschr. 1916 Nr. 17), von I. Pick (Dtsch. med. Wochenschr. 1916, Nr. 33), sowie von mir (Beitr. z. pathol. Anat. u. z. allg. Pathol. 63, $60 \mathrm{ff}$.) ïbcreinstimmend gelehrt. Während Benda innerhalb jeder in den verschiedensten Schichten der Lederhant, mit Ausschlul3 der Papillen, gelegenen Blutung einen kleineren oder größeren, vorwiegend aus polynucleären Leukocyten bestehenden, spärliche Mast-und Lymphzellen enthaltenden Entzündungsherd findet, bemerkt Pick, daß diese entzündlichen Prozesse nicht selten unabhängig von Austritt der Erythrocyten bestchen, erwähnt auch, daß er, abweichend von Benda, den Papillarkörper nicht regelmäBig frei von Blitt gefunden hat. Benda führt, wenigstens für 2 seiner 3 untersuchten Fälle, das Vorkommen von teils hyalinen, teils zelligen Thromben in den Arterien an, ein Ereignis, das Pick wiederum in seinen beiden Fällen vermißt hat. Ich hake in meinen bis zum Jahre 1916 untersuchten 4 Fällen 2 mal einen bis dahin unbekannten Befund erhoben, den ich als Arterionekrose bezeichnet habe. Die so schwer geschädigte Arterie war das eine Mal von einem einfachen, in seiner unmittelbaren Nachbarschaft gelegenen Extravasat, das andere Mal, bei dem ein größerer in der 
Subcutis gelegener, schon mit dem bloßen Auge erkennbarer Arterienast ergriffen war, von einem ausgesprochen entzündlichen Exsudat, mit Thrombierung der im halben Umfang nekrotischen Arterie, begleitet. In dem dritten meiner Fälle wurde wiederum ein reines Extravasat am Übergang der P. retic. in den Papillarkörper in unmittelbarer Umgebung eines präcapillaren Ästchens nachgewiesen, das an einer Stelle durch einen langgestreckten hyalinen Thrombus nahezu völlig verschlossen war. In dem letzten Fall endlich bestand, in dem einen der 2 untersuchten Hautstückchen, eine komplette Capillarstase, ohne jede anderweitige Veränderung, während in dem anderen eine leukocytäre Verstopfung einer Präcapillare und leukocytäre Infiltration ihrer Wand und der unmittelbarsten Umgebung festgestellt werden konnte. Auch Benda hat am GefäBapparat, ebenso wie ich, hyaline und zellige Thromben, sowie entzündliche Infiltrate der Gefäßwand beobachtet. Endlich habe ich 2 mal eine, die gesamte Dicke der Wand, wenn auch nicht den ganzen Umfang der befallenen Gefäßstrecke betreffende, Nekrose mit völliger Auslöschung der Struktur an dem geschädigten Gefäßstück feststellen können. Diese Fülle der histologischen Befunde an den bei manchen Fällen epidemischer Genickstarre auftretenden Hautpetechien hat $\mathrm{L}$. Pick scharf betont und dabei bemerkt, daß, die feineren histologischen Befunde kei der petechialen Form des die Mgt. epidem. begleitenden Exanthems keine Einheitlichkeit aufweisen und sich vielleicht auch nicht einmal in den bisher bekannten Einzelheiten erschöpfen." Eine Bestätigung dieser Pickschen Ansicht bringt die nachstehende, beiläufig als einzige von mir seit dem Jahre 1916 gemachte Beobachtung eines petechialen Exanthems bei epidemischer Genickstarre.

Es handelt sich um einen 5 jährigen Knaben Br., der am 19. III. 19 aufgenommen und am 18. IV. 19 geheilt entlassen wurde. Auf der Haut der Brust. und des Bauches, der Arme und des Gesichts (sowie der Conjunct. bulb.) fanden sich bei dem, 4-5 Tage vor der Aufnahme mit Übelkeit, Kopfschmerzen und Erbrechen erkrankten Knaben zahlreiche kleine, punktförmige, bis pfenniggroße Blutungen; es bestand leichte Nackensteifigkeit. Die Lumbalpunktion ergab erhöhten Druck und tr übe $n$ Li qu or. Im Zentrifugat intracellulär gelegene, gramnegative Diplokokken. In der Rekonvaleszenz wurden, bis über den 2. April hinaus, auch im Rachenabstrich gramnegative Kokken nachgewiesen. Der kulturelle Nachweis der Krankheitsrerger im Blut gelang, obwohl in die verschiedensten festen und flüssigen Nährboden verimpft wurde, nicht. Am Tage der Aufnahme wurde eine kleine Petechie herausgeschnitten und nach Fixierung in Müller-Formol in Serienschnitte zerlegt; Färbung mit Eosin-Hämotoxylin, mit Orcein-Meythlenblau und nach Pappenheims panoptischer Methode. Dabei ergab sich folgendes:

Es finden sich teils am Übergang des Corium in das subcutane Fettgewebe, und zwar hier innerhalb der sich von der Lederhaut in die Subcutis erstreckenden bindegewebigen Ausläufer, teils frei im Fettgewebe gelegene, streifige und rundliche, zellig-hämorrhagische Herde, in denen bei einzelnen die hämorrhagische, 
bei andern die leukocytäre Komponente überwiegt. Sie sitzen stellenweise in der Umgebung arterieller und venöser Äste, bisweilen auch unabhängig von solehen. Unter den farblosen Zellen ïberwiegen bei weitem die polynucleären, denen gegenüber proliferierte, fixo Gewebszellen in den Hintergrund treten. Sämtliche Herde sind nur klejn, der größte, halbkugelige sitzt ain Übergang dor Cutis in dic Unterhaut; zahlreiche kleinere in Fettgewebe, zwischen den einzelnen Fettzellen. An den auf dic Darstellung des elastischen Gowebes gefärbten Schnitten sicht man in dem größten, zwischen einem dickeren Arterienast und dem sie begleitenden Nervenstämmchen gelegenen Herd, annähernd in dessen Zentrum, eine umschriebene, schmutziggrau tingierte, anscheinend nekrotischem, kollagenem Gewebe entsprechende itelle, innerhalb deren auch die bis clahin deutliehen, clastischen Fasern unsichtbar worden. Die Wandungen der in der Cutis wie Subcutis verlaufenden Gefïße erweisen sich vollkommen intakt. Auch ihr Lumen ist frei von abnormem Inhalt, spez. von Krankheitserregern. Dagegen gelang es, solche, freilich auch nur vereinzelt, und zwar, soweit din Urteil möglich, intracellular gelagert, als Diplokokken festzustellen.

Es ist durch diesen Befund der metastatische Charakter der Hautpetechien einwandfrei dargetan. An dieser Auffassung des petechialen Exanthems bei der epidemischen Genickstarre ist seit dem durch Benda und Pick im Jahre 1916 erbrachten, wichtigen

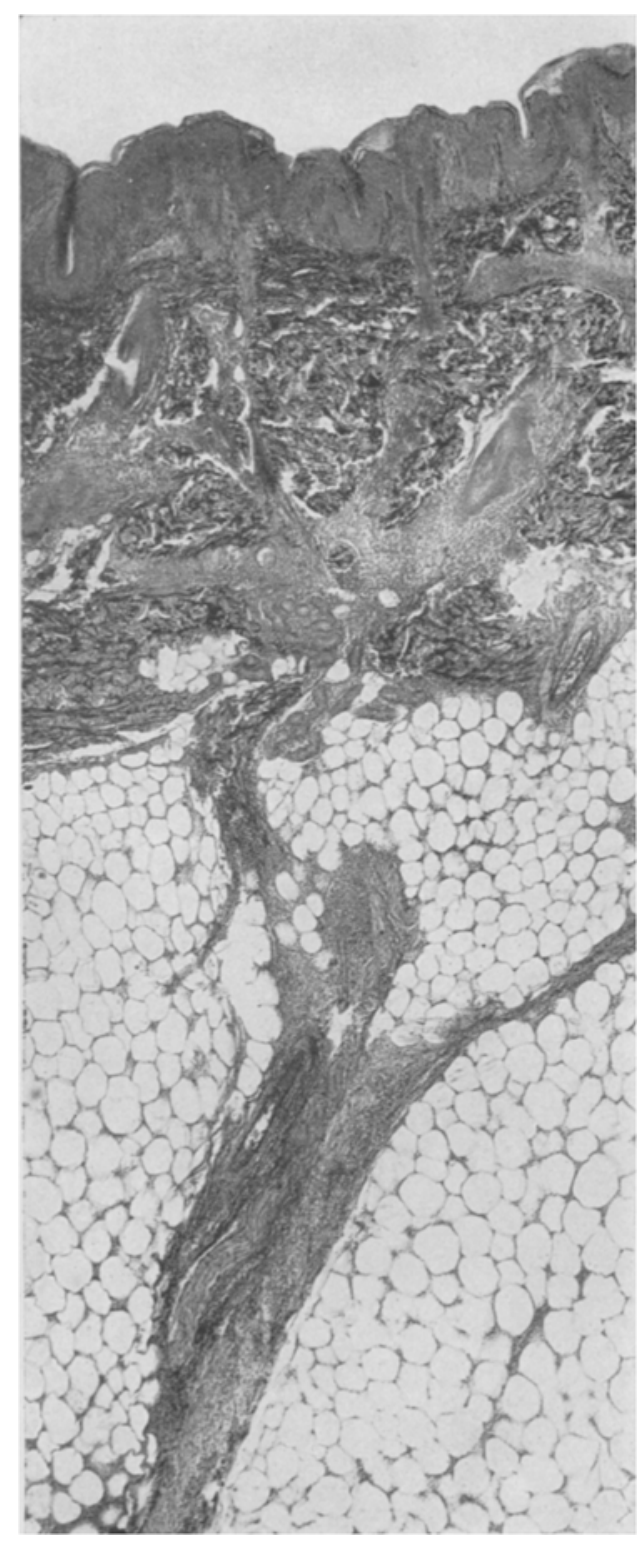

Abb. 1. Übersichtspräparat (Elastica-Färbung). Hämorrhagiseh-entzindlicher Hantherd bei MenirigokokkenMeningitis. 
Nachweis der Krankheitserreger in einzelnen, zum Gebiet des Blutherds gehörenden Arterienästchen nicht mehr zu zweifeln; besonders die von Pick seiner Arbeit beigegebenen Photogramme der, Meningokokken in. größerer Zahl enthaltenden Arterien sind in dieser Beziehung ebenso lehrreich wie überzeugend. In dem vorliegenden Fall fanden sich die Erreger nicht, oder wahrscheinlich nicht mehr, intravasculär, sondern innerhalb eines der die Subcutis in größerer Zahl durchsetzenden, zelligen Exsudate. Aber es ist ganz selbstverständlich, daß sie dahin erst von der Gefäßbahn aus gelangt sind. Thr Auffinden war mit großer Mühe verknüpft. Es ist wahrscheinlich, daß, wenn die Ausschneidung des erkrankten Hautstückes 24 Stunden später erfolgt wäre, das Suchen nach Meningokokken keinen Erfolg mehr gehabt hätte, weil die ohnehin nur in geringer Zahl in dem entzündlichen, zelligen Exsudat abgelagerten Krankheitserreger dann dem Untergang verfallen gewesen wären. Vermutlich erfolgt ihre Vernichtung, wie Pick l. e. betont, sehr schnell, schon innerhalb der Blutbahn, und darauf ist es wohl zurückzuführen, wenn ihr Nachweis in den petechialen Hautherden bei der epidemischen Genickstarre nur ausnahmsweise und mit einiger Aussicht auf ein positives Ergebnis anscheinend nur dann gelingt, wenn es sich um besonders rapid, innerhalb 24-48 Stunden, zum Tode führende Fälle handelt. Sind die Meningokokken erst einmal aus den Gefäßen in das umgebende Gewebe gelangt, und haben sie dort zur Bildung reaktiver Veränderungen in Gestalt zelliger, hämorrhagischer oder nicht hämorrhagischer Herde geführt, dann sind die Chancen, sie anzutreffen, erst recht gering. Einmal, weil sie in den Zellanhäufungen schwer sichtbar zu machen sind, und ferner, weil sie dort wohl rasch der Auflösung verfallen.

Jedenfalls lehrt der hier mitgeteilte Fall, daß man bei de m Suchen nach Meningokokken in Hautpetechien nicht bloß a uf die zu den Entzündungsherden führenden Arterienästchen, sondern auch auf die sie zusammensetzenden, zelligen Elemente zu achten hat. Auch in histologischer Beziehung hat der vorliegende Fall eine bis dahin nicht beobachtete Tatsache zutage gefördert, ich meine die umschriebene, kollagenes Gewebe und anscheinend auch elastische Fasern betreffende Nekrose in einem der geschilderten Entzündungsherde.

Wir hätten also bis jetzt, neben einfachen und mit entzündlichen Prozessen vergesellschafteten Extravasaten in der Haut Genickstarrekranker, auch das Vorkommen von umschriebenen Nekroseherdchen bei den petechialen Hautaffektionen zu buchen und hinsichtlich der Lokalisation der Krankheitserreger, neben ihrem intravasculären Aufenthalt, ihr Vorkommen in entzündlichen Infiltraten zu erwähnen. 
Für das bloße Auge handelt es sich in dem einen wie dem andern Fall um einfache Extravasate.

Bemerkenswert ist der negative Ausfall der Blutkultur. Wenn sie in diesem wie in zahlreichen anderen Fällen von Genickstarre versagt hat, so ist der Grund hierfür in der von Pick gegebenen Erklärung zu finden, daß sie eben schnell a us der Blutbahn verschwinden, weil sie, wahrscheinlich schon innerhalb derselben abgetötet, in unseren Nährböden nicht mehr zur Entwicklung gelangen.

In klinischer Hinsicht ist darauf hinzuweisen, daß auch dieser Fall in Genesung übergegangen ist und somit eine Bestätigung der von Benda gemachten, durch eine meiner früheren Beobachtungen bewahrheiteten Angabe liefert, daB Petechien auch bei klinisch leichten Fällen von epidemischer Genickstarre auftreten können. Aber im allgemeinen trifft das nicht zu; in diesem Sinne reden die Mitteilungen von Benda, Pick und mir eine sehr deutliche Sprache.

Alles bisher Gesagte bezieht sich

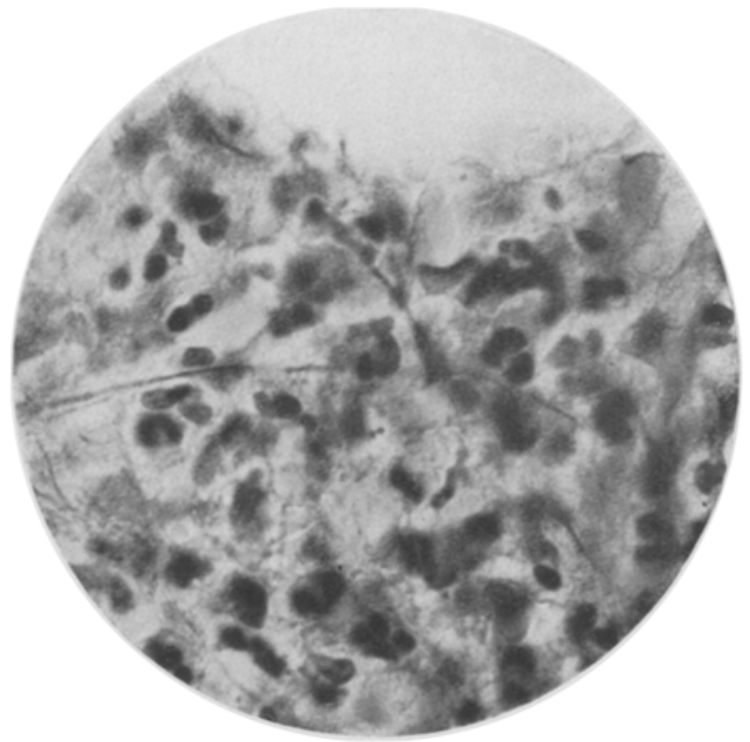

Abb. 2. Ein dentliches Moningokokkenpanr frei im zelligen Exsudat. lediglich auf die im Verlauf der epidemischen Genickstarre auftretenden petechialen Hauterkrankungen, Über die verschiedenen anderen Exanthemformen, die, wie masern-oder urticariaähnliche, oder wie die unter dem Bilde des Erythema nodosum sich präsentierenden $\Lambda$ usschläge, bei dieser Krankheit beobachtet werden, wissen wir hinsichtlich des ihnen zugrunde liegenden anatomischen Substrats nicht das geringste. Und ebensowenig vermögen wir darüber etwas auszusagen, ob sie a uf eine direkte Invasion der Meningokokken in die Haut zurückzuführen, also als infektiöses Fxanthem aufzufassen sind, oder ob sie toxischen, durch den Meningokokkus im Körper erzeugten Substanzen ihre Entstehung verdanken. Hier bleibt also der Forschung noch ein weites und dankbares Feld. 
Ich reihe hier die Besprechung eines anderen Falls von eitriger Meningitis an, bei dem nicht Meningokokken, sondern Friedländerbacillen als Erreger in Betracht kommen. Die Beobachtung ist insofern etwas lückenhaft, als eine vollständige Sektion fehlt. Wir mußten uns, da eine solche verweigert war, auf die Herausnahme des Rückenmarks beschränken. Dabei ließ sich ein, besonders an der

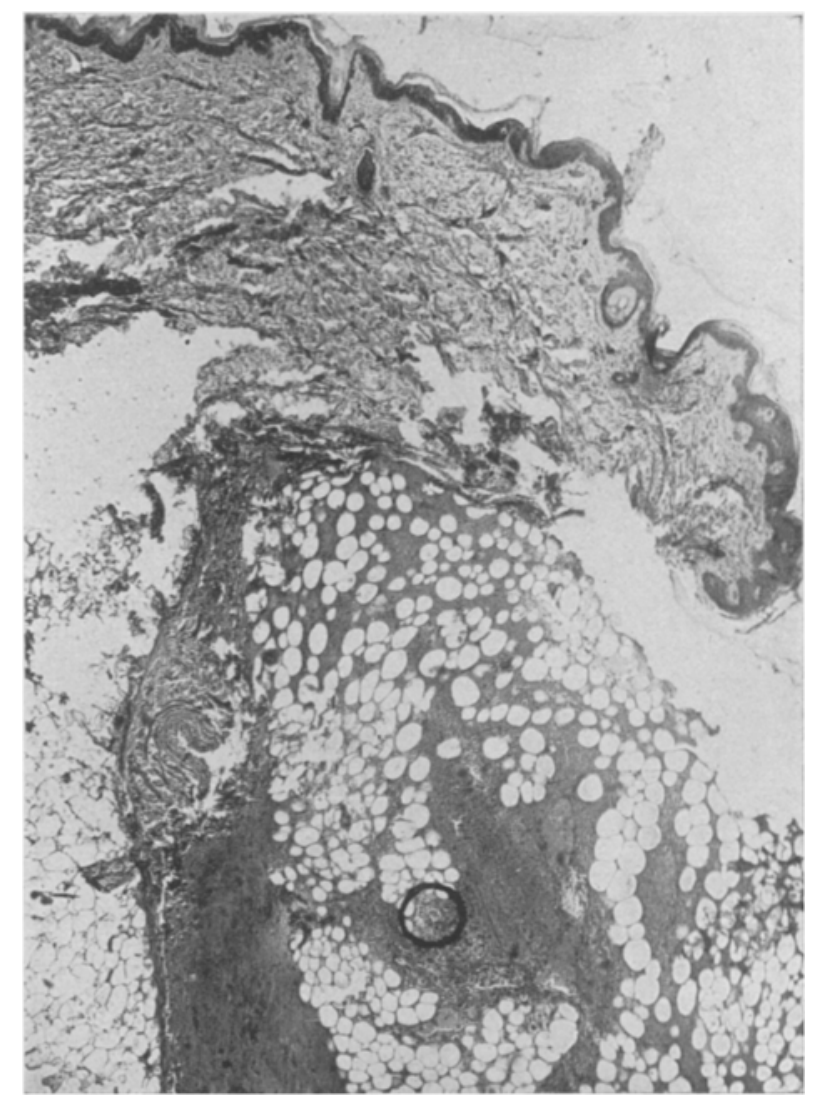

Abb. 3. Hämorrhagisch-entzündlicher Herd der Subeutis bei Meningitis durch Friedländerbacillen.

Hinterfläche des Dorsalteils deutliches, trübseröses Exsudat feststellen, aus dem mittels Blutplattenkultur Friedländerbacillen gezüchtet wurden. Genau das gleiche Ergebnis war am Krankenbett durch Aussaat der durch Lumbalpunktion gewonnenen Cerebrospinalflüssigkeit auf Blutagar erzielt worden. Außerdem zeigte ein direkter Ausstrich des Sediments dieser Flüssigkeit auf einen Objektträger ziemlich zahlreiche gramnegative Stäbchen. 
Bei der äuBeren Besichtigung der Leiche dos 5jährigen Mädchens fielen mir sofort an der Haut beider Unterschenkel mehrere livide, bis zehnpfennigstückgroße Flecke auf, denen in der Subcutis sitzende dunkelrote Extravasate entsprachen. Von zweien dieser Blutherde wurde unter den üblichen Kautelen Material auf je eine Agar- bzw. Blutagarplatte übertragen. Nur an einer Stelle der letzteren entwickelte sich im Bereich eines auf dem Nährboden befindlichen Stückehens Fettgewebe eine Friedländerbacillenkolonie. Dadurch war die auf Verschleppung des Erregers der Meningitis zurückzuführende, also hä matogene Entstehung der hämorrhagischen Herde in der subcutis bewiesen. Fis blieb noch übrig, histologisch zu prüten, ob hier tatsächlich, wie (lic makroskopische Betrachtung zu zeigen schien, einfach hämorrhagische ofler komplizicrte Prozesse vorlaggen.

In Ċbercinstimmung mit der makroskopischen Deutung Jehrte das Mikroskop, lab sich die Veränderungen ausschlieblich auf die Subcutis beschrïnken. Man sjeht hicr ein in seiner Form

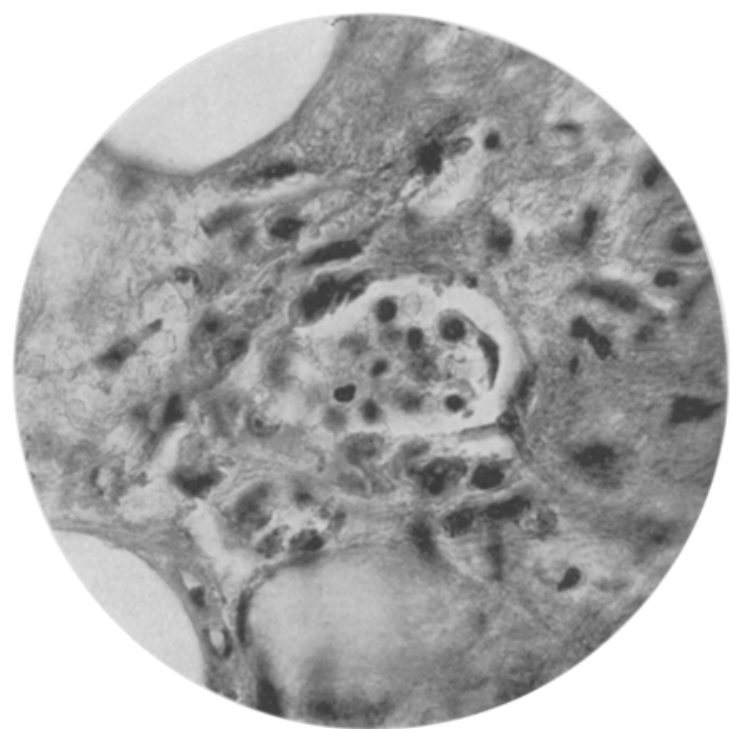

Abb. 4. Blutgetäls mit Leukocyterithrombus; in der ciefäswand eil polgefarbter liriedländerbacillus: starko Vergröberung aus dem umrandeter Bozirk der Abb. 3. zunächst streifiges, allmählich, mit der Spitze gegen die Haut gerichtetes, Keilform annehmendes, schließlich kugelig werdendes Extravasat, das in den gröberen und foineren, die einzelnen Fetträubchen trennenden, bindegewebigen Septen sitzt. An versehiedenen Stellen sind ihm, in zum Teil beträchtlicher Menge, lange, spindelige, eckige, sternförmige Zellen mit chromatinreichem Kern beigemengt. Leuko- und Lymphocyten fehlen. Die Zellen finden sich vielfach in unmittelbarster Umgebung von Arterienästchen mit deutlich geschwollenen Endothelien. An einem dieser GefäBe gelingt der Nachweis eines extravasculär gelegenen, deutliche Polfärbung a ufweisenden stäbchens.

Die mikroskopische Untersuchung ergänzt in verschiedener Richtung das Resultat der bakteriologischen Feststellungen, einmal durch den 
gelungenen Nachweis des Krankheitserregers in dem erkrankt befundenen Gewebe und ferner dadurch, daß sie zeigt, daß es sich nicht, wie man nach Besichtigung mit bloßem Auge annehmen durfte, um eine einfache blutige Durchsetzung der Subcutis, sondern um eine hämorrhagische Entzündung mit Alteration einzelner Arterienästchen gehandelt hat. Es liegt, wie bereits erwähnt, ein metastatischer Vorgang in der Haut vor, der den bei der Meningokokkenmeningitis beschriebenen petechialen Hautaffektionen in Parallele $\mathrm{zu}$ setzen ist. Seitdem Benda und Pick bei diesen die Krankheitserreger, in zum Teil großer Menge, histologisch nachgewiesen haben, sind diese Befunde von anderen Beobachtern insofern bestätigt worden, als ihnen der Meningokokkennachweis in den Petechien durch das sehr viel bequemere Züchtungsverfahren gelungen ist (vgl. Centralbl. f. Bakt. u. Parasitenk. Ref. 69, Nr. 7/8, 177, 178).

Angaben über das Vorkommen von petechialen Vorgängen in der Haut bei eitriger Meningitis durch andere Erreger fehlen vollständig. Es ist das wohl, zum Teil wenigstens, auf die sehr viel geringere Häufigkeit dieser Meningitisfälle zurückzuführen. Werden doch die sog. Friedländerpneumonien, im Gegensatz zu den Pneumokokkenpneumonien, kaum jemals durch auf den gleichen Erreger zurückzuführende Hirnhautentzündungen kompliziert. Bei dem hier in Rede stehenden Fall bestanden keinerlei Erscheinungen seitens der Lunge, und bei dem Fehlen einer vollständigen Sektion ist die Frage nach der Eingangspforte für den Krankheitserreger, der hier zur eitrigen Meningitis geführt hat, nicht $\mathrm{zu}$ beantworten. Aber daran ist nicht zu zweifeln, daß, zumal Nase und Ohren des Kindes, von denen aus durch Fortleitung eitrige Hirnhautentzündungen entstehen können, ausdrücklich als intakt bezeichnet worden sind, die eitrige Meningitis sowohlals a uch die Hautaffektion als hämatogenentstanden anzusehen sind.

Ich habe früher einmal eine schwere metastatische Hautaffektion im Anschluß an eine Allgemeinerkrankung durch den sog. Friedländerbacillus beobachtet und darüber in der zum 60. Geburtstag des Jubilars erschienenen Festschrift (S. 84) berichtet. Sie unterschied sich von den hier multipel aufgetretenen Unterhautherden schon dadurch, daß nur ein einziger, klinisch als Furunkel gedeuteter Herd vorlag, bezüglich dessen feinerem Bau ich auf die 1. c. gegebene Schilderung verweise. In einer Hinsicht besteht eine gewisse Ửbereinstimmung insofern, als dort wie hier sich der Krankheitsprozeß in der Subcutis abspielte. Während aber dort die Bacillen sich in großer Menge und in dichten Schwärmen im Gewebe angesiedelt hatten, gelang es hier nur mit Mühe, in einem Schnitt einer Serie einen Bacillus aufzufinden. 
Ich bemerke übrigens, daß mit der Bezeichnung Friedländerbacillus nicht ohne weiteres gesagt sein soll, daß die in beiden Fällen aufgefundenen Bacillen tatsächlich als identisch anzusehen sind. Man ist m. E. nur berechtigt, von Bacillen der Friedländerg r u p pe zu sprechen. Es kommen, namentlich in bezug auf die bei den einzelnen Stämmen dieser Gruppe vorhandenen schleimigen Hüllen, außerordentliche Verschiedenheiten vor, und es erscheint mir zweifelhaft, $o b$ man berechtigt ist, die verschiedenen Arten dieser Gruppt', wie den Abelschen Ozaenabacillus, einen von mir vor Jahren als B. mucos. capsulat. und den eigentlichen Friedländerbacillus, der keineswegs ein schleimiges Wachstum aufweist, sämtlich für identisch zu crklären. Der im vorliegenden Fall gefundene Bacillus bildete auch keine ausgesprochen schleimigen Kolonien, entsprach aber übrigens der scinerzeit von dem Entdecker für scinen Bacillus gegebenen Schilderung.

Úber Hautaffektionen bei Allgemeinerkrankungen durch den Friedländerbacillus liegen Angaben überhaupt nicht vor. Selbst der neneste Bearbeiter des Kapitels Sepsis (Kraus-Brugsch, Spez. Pathologie und Therapie innerer Krankheiten, Lieferung 92/93, S. 1108 ff.) Leschke beschränkt sich darauf anzuführen, daß er in einem Fall von „Friedländer-Sepsis“ mehrere subcutane Abscessc beobachtet hat, erwähnt das Vorkommen von petechialen oder nicht petechialen Prozessen in der Haut überhaupt nicht. Seine Darstellung rleckt sich übrigens fast wörtlich mit der von Jochmann in seinem vortrefflichen Lehrbuch der Tnfektionskrankheiten vier Jahre früher gegebenen. Dort heilst es \$. 173: „Klinisch verläuft die TriedländerSepsis teils mit, teils ohne eitrige Metastasen", bei Lesch ke: ,Der Verlauf cler Friedlander-Sepsis kann sowohl metastasierend, als auch nicht metastasierend sein." Und weiter bei Jochmann: ,Endokarditis, Perikarditis und multiple Gelenkeiterungen, subcutane Hauteiterungen und miliare Nierenabscesse werden dabei beobachtet." Bei Leschke: „Endokarditis, Perikarditis, Gelenkeiterungen, Nicrenakscesse, Menin gitis sind beobachtet worden." In der Tat eine geradezu überwältigende Úbereinstimmung nicht nur der Ansichten, sondern auch der Worte des Herrn Leschke mit denen von Jochmann.

Ich glaube, für die von mir beobachteten, bei schweren Allgemein affektionen durch sog. Friedländerbacillen verursachten Haut. affektionen den Beweis erbracht zu haben, daß die Wirkung dieser Bacillen a uf das Haut-, richtiger Unterhautgewcbe eine ganz verschiedene sein kann, und daß, entsprechend dem wechselnden makroskopischen Verhalten, auch weitgehende histologische Differenzen zu konstatieren sind. Während bei dem in der ersten Unna-Festschrift publizierten Fall die Schnittfläche durch den erkrankten Hautbezirk einen zähschleimigen. Inhalt austreten ließ, und sich der Prozeß schon 
dadurch als ein ganz eigenartiger charakterisierte, haben wir hier makroskopisch das Bild einfacher Unterhautblutungen, das durch die mikroskopische Untersuchung eine wesentliche Modifikation erfährt und die Affektion als eine entzündliche erkennen läßt.

Bei der bisherigen Spärlichkeit der Befunde lassen sich weitere Schlußfolgerungen über die Wirkung der sog. Friedländerbacillen auf das Hautorgan nicht ziehen, und es bleibt fortgesetzten Untersuchungen vorbehalten, festzustellen, ob die genannten Krankheitserreger noch anderweitige als die jetzt nur durch mich bekannt gewordenen Ver änderungen an der Haut auszulösen vermögen. In klinischer Beziehung erscheint es mir wichtig, die durch den mitgeteilten Fall zum erstenmal erhärtete Tatsache zu betonen, daß a uch bei anderen, als durch Meningokokken verursachten, eitrigen Hirnhautentzündungen petechiale $Z$ ustände in der Ha ut herbeigeführt werden können. Freilich unterscheiden sich diese von den bei der Meningokokken-Meningitis vorkommenden, fast ausnahmslos das eigentliche Hautgewebe betreffenden kleinen und kleinsten hämorrhagischen Fleckchen einmal durch ihre Größe und vor allem durch ihren ausschließlichen Sitz im Unterhautfettgewebe. Grade dadurch dürften sie auch zu Verwechslungen mit Fleckfieber, wie sie namentlich in Zeiten von Fleckfieberepidemien durch die, manche Fälle von MeningokokkenMeningitis begleitenden, petechialen Exantheme vorgekommen sind, nicht den geringsten Anlaß geben.

Ganz und gar lückenhaft sind unsere Kenntnisse über Erkrankungender Haut beidurch Pneumokokken hervorgerufenen Allgemeininfektionen. Freilich muß hervorgehoben werden, daß dabei die Haut nur ganz ausnahmsweise in Mitleidenschaft gezogen zu werden scheint. In der ausgezeichneten Monographie von Lenhartz „Über septische Erkrankungen" habe ich ebensowenig wie in dem. „Lehrbuch der Infektionskrankheiten" des leider so früh verstorbenen Jochmann irgendwelche Angaben über das Vorkommen von Erkrankungen der Haut bei "Pneumokokkensepsis" gefunden. Es ist das um so auffallender, als wir nicht bloß bei schweren, tödlich verlaufenden, sondern auch bei leichteren akuten, durch den Pneumokokkus verursachten Allgemeininfektionen, vor allem bei der durch diesen erzeugten Pneumonie, Metastasen in den verschiedensten Organen beobachten. Ich sehe dabei von der nahezu ausnahmslos zum Tode führenden schwersten Komplikation durch eine Meningitis, oder der regelmäßig letal endenden Endokarditis ab, und erwähne metastatische Prozesse in den Gelenken und den Umbüllungen des Hodens. Die Ha u t scheint sich demgegenüber einer gewissen Im munität zu erfreuen. Denn da die Krankheitserreger, wie die Blutkultur beweist, oft in außerordentlich großer Menge im Blut kreisen, müssen sie notwendig 
auch in die Haut verschleppt werden. Aber selbst wenn die Blutplatte mit ihnen überladen ist, scheinen sie in der Haut nicht festen Fuß fassen zu können, sondern dort so rasch zugrunde zu gehen, daß sie das Gewebe nicht zu schädigen imstande sind. In dieser Beziehung nimmt der Diploc. lanz. keine Sonderstellung ein. Wir kennen das gleiche Verhalten der Haut auch anderen Bakterien gegenüber. Ich erinnere nur an den Tuberkelbacillus, der, obwohl bei der akuten Miliartuberkulose das Blut zeitweise große Mengen Tuberkelbacillen enthält und dadurch zur Entstehung massenhafter Knötchen in fast sämtlichen Organen des

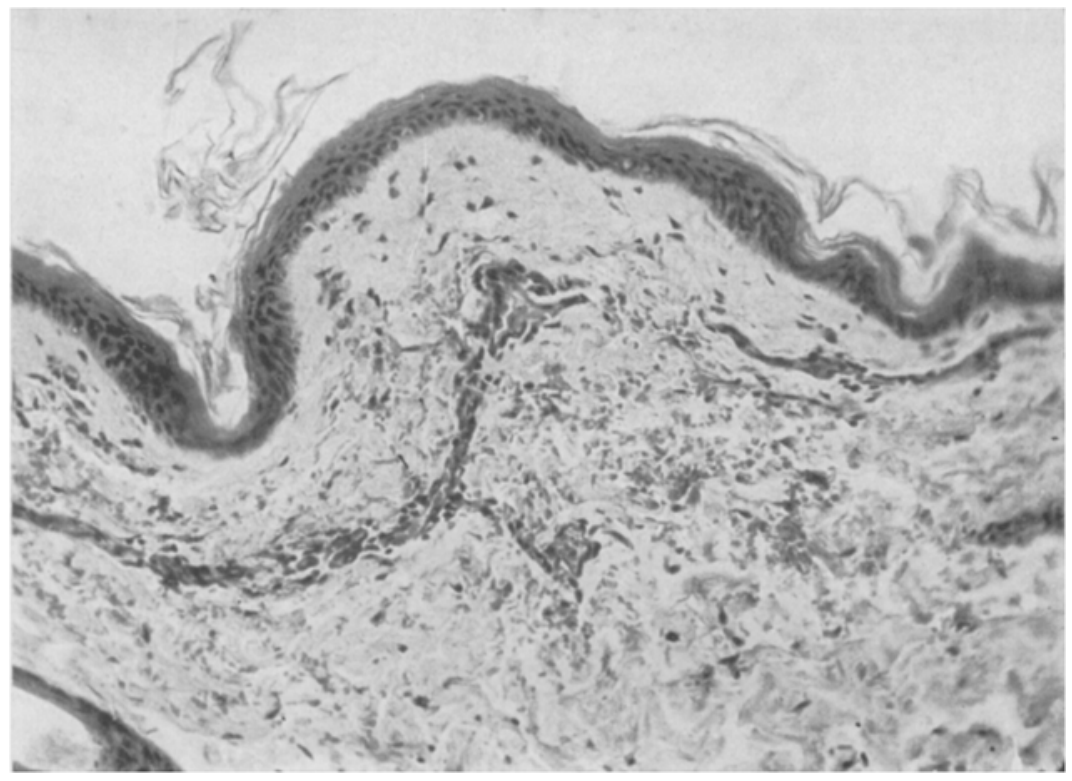

Abb. 5. Hautbluturig in der P. reticulari bei Plienmokokkätuie.

Körpers Veranlassung gegeben ist, in der Haut nur in den allerseltensten Fällen einen ährlichen Prozeh auslöst.

Für den Diploe. lanz. wissen wir äber lie Folgen dureh direktes Eindringen in die Haut erzeugter Erkrankungen überhaupt nichts und über etwaige, auf hämatogenem Wege erfolgte Infektionen so gut wie nichts. Deswegen möchte ich über zwei eigene, hierher gehörige Boobachtungen berichten.

Im ersten Fall handelt es sich um cine 34 jährige, an schwerster Pnemmokokken-Allgemeininfektion zugrunde gegangene Frau, bei der sich die Erkrankung an einen Abort im 2./3. Monat angeschlossen hatte. Die am 11. VI. 19 aufgenommene Patientin ging am 2I. XI. zugrunde. Sie war bei der Aufnahme schwach ikterisch, der Ikterus schwand aber während des Krankenhausaufenthalts. An 16. XI. stellte sich Narkensteifigkeit cin. Im Lumbalpunktat wurlen 
Pneumokokken nachgewiesen, nachdem die Blutkultur schon bei der Aufnahme die Anwesenheit von solchen festgestellt hatte, und zwar fanden sich in $2 \mathrm{ccm}$ 66 Pneumokokkenkolonien. Bei einer am 16. XI. erneut vorgenommenen Blutkultur waren die Agarplatten durchsetzt von Pneumokokken. Bei einer letzten, am Todestage der Patientin vorgenommenen Blutuntersuchung enthielt $1 \mathrm{ccm}$ Blut unzählige Pneumokokkenkolonien. Am 19. XI. erwähnt die Krankengeschichte zum erstenmal , an der Haut des Rumpfes, bes. zahlreich des Rückens, spärlicher an den Extremitäten, dagegen dicht am Hals und der Stirn feine, stecknadelspitzen-, -kopf-, vereinzelt auch linsengroße, unregelmäßig begrenzte Blutungen, auch auf der Conjunctiva und der Wangenschleimhaut, die wie mit Blutspritzern bedeckt ist". Am 20. XI. wird über stecknadelkopfgroße Roseolen mit stippchenförmigen gelben Zentrum berichtet.

Die klinische Diagnose lautet auf Endokarditis mitral. durch Pneumokokken nach Abort, metastatische Nierenabscesse, Milzinfarkte, eitrige Meningitis.

Es war nur eine Teilsektion gestattet, welche die klinische Diagnose vollkommen bestätigte. Von der Schädelsektion mußte allerdings abgesehen werden. An der Haut der Leiche ließen sich nur die geschilderten kleinen Blut. a ustritte, nicht aber die am 20. XI. erwähnten ,Roseolen mit stippchenförmigem Zentrum" nachweisen. Ich mußte mich auf die histologische Untersuchung einer kleinen Petechie beschränken, die folgendes ergab:

Man erkennt am Übergang des Papillarkörpers in die P. reticul. wenig dicht zusammenliegende Erythrocyten, die eine hier verlaufende, sich gablig teilende Präcapillare einigermaßen umscheiden. Entzündlich-exsudative Veränderungen fehlen, die der Gefäßwand außen anliegenden Zellen erscheinen stellenweise etwas geschwollen, bes. ihre Kerne, während die Intima mit ihren Endothelien nicht verändert ist. Einer Seite der Wand sitzt ein das Lumen etwas einengender, leicht krümliger Thrombus auf; das in die Cutis gesetzte Extravasat läßt sich durch 8 Schnitte verfolgen. Mikroben finden sich weder intravasculär noch frei im Gewebe. Die Oberhaut ist völlig normal.

Auch mikroskopisch hat es sich also um einen ganz reinen Blutaustritt gehandelt, der um einen, durch einen wandständigen Thrombus in seinem Lumen leicht eingeengten Gefäßast gelegen ist. Es wirft sich nun die Frage auf, ob hier in der Tat ein mit der schweren Allgemeininfektion zusammenhängender Vorgang zu erblicken ist, und diese möchte ich bejahen, obwohl das Argument, das als in dieser Beziehung sicher beweisend anzusehen gewesen wäre, die Anwesenheit der dem Allgemeinleiden zugrunde liegenden Krankheitserreger, nicht vorliegt. Aber hier darf man wohl Analogieschlüsse ziehen, und da möchte ich gerade auf die bei der epidemischen Genickstarre gemachten Beobachtungen hinweisen, bei der gleichfalls in den Hautpetechien hyaline Thromben gefunden worden sind. Diese sind zweifellos auf eine Schädigung der Gefäßwand zurückzuführen, die in dem hier mitgeteilten Fall zudem in der Schwellung einzelner, der Gefäßwand anliegender bindegewebiger Zellen zum Ausdruck gekommen ist. Es liegt durchaus nahe, sich vorzustellen, daß für das Zustandekommen der, wenn auch geringfügigen, Gefäßwandschädigung die in die Haut eingeschwemmten Krankheitserreger verantwortlich zu machen sind. Ihr Fehlen zur Zeit der Untersuchung spricht durchaus nicht gegen die 
hier vorgetragene Amnahme, wie ebenfalls die an den petechialen Hautausschlägen bei der epidemischen Genickstarre gemachten Erfahrungen lehren.

Der 2. Fall betrifft einen, am. 23. H. aufgenommenen, am 26. II. verstorbenen 21 jährigen Mann, der 5 Tage vor der Aufnahme mit Heiserkeit, Kopfschmerzen, Kratzon im Hals, Brustschmorzen und Husten erkrankt und am Tage

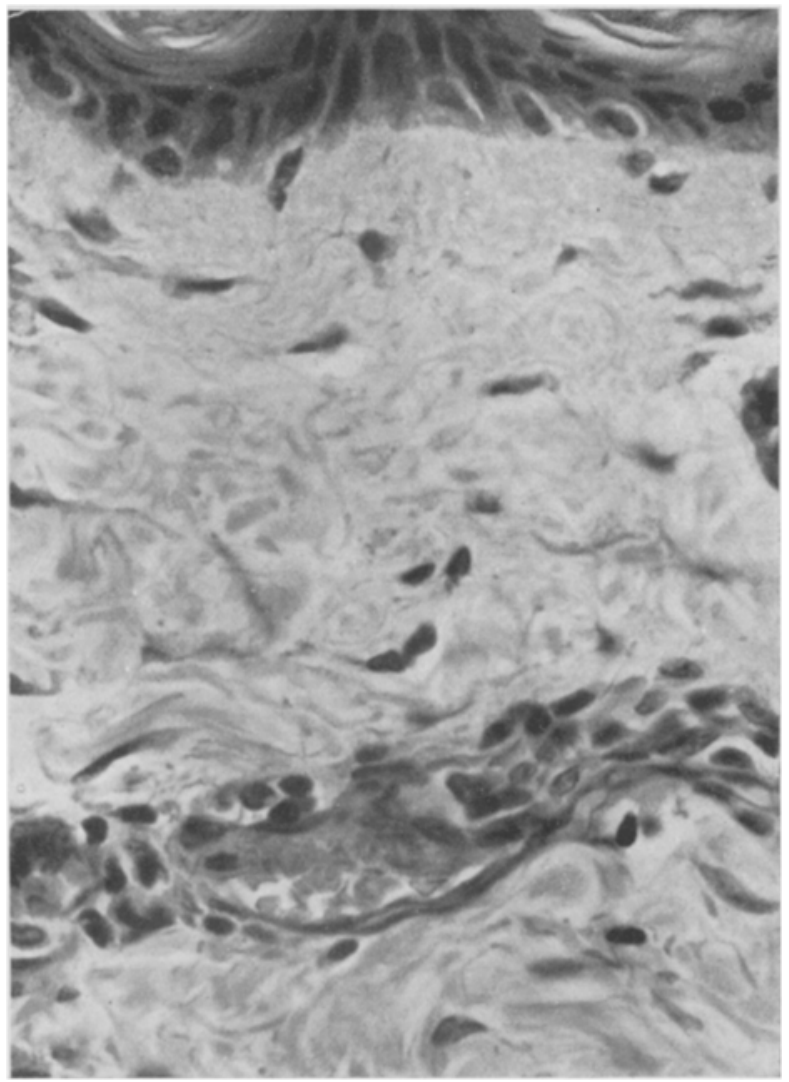

Abb. f. Krïmliger, wandständiger Tlrombus ì einer Präcapillare der P. reticularis.

der Aufnahme gelb geworden war. Im Krankenhause wurde eine Pneumonie des r. Unterlappens festgestellt. Der Ikterus nahm zu, es traten Durchfälle ein, am 25. II. machte sich Nackenstarre bemerkbar, gleichzeitig sah man zahlreiche kleine Hautblutungen, hauptsächlich am Bauch und an den Flanken. Unter zunchmender Benommenheit starb Pat. am 26. Die klinische Diagnose lautete: Pneumonie des r. Unterlappens, Bakteriamie durch Diploc. lanz., Ikterus, Hautblutungen, Meningitis.

Bei der Sektion des $182 \mathrm{~cm}$ l. ikterischen, mageren Mannes wurde die klini . sehe Diagnose bestätigt; die Leber war deutlich verfettet, die Gallenwege 
waren völlig durchgängig. An der Haut des Bauches feinste, punktförmige Blutungen, ebensolche auch an der Hinterfläche des Epikard.

Zur mikroskopischen Untersuchung gelangten 2 der erwähnten Hautblutungen. Überraschenderweise fand sich nun Blutaustritt ins Gewebe überhaupt nicht, sondern nur eine starke Füllung der Capillaren und Präcapillaren und in einer der letzteren ein hyaliner, streckenweise obturierender Thrombus. Außerdem zeichnete sich dieser ganze Bereich der Cutis durch einen auffallenden Reichtum an Zellen aus, die sämtlich als Abkömmlinge fixer Gewebszellen anzusehen sind. In dem unteren Ast der Gabel enthält das hier an einer Stelle eröffnete Lumen einen nahezu reinen Leukocytenthrombus. Der ganze Prozeß ist, entsprechend den als feinste punktförmige Blutaustritte imponierenden Herdchen, auf etwa 6 nebeneinander liegende Papillen beschränkt.

Der hier mitgeteilte weicht von dem im vorigen Fall erhobenen Befund insofern wesentlich ab, als hier an den als Petechien gedeuteten

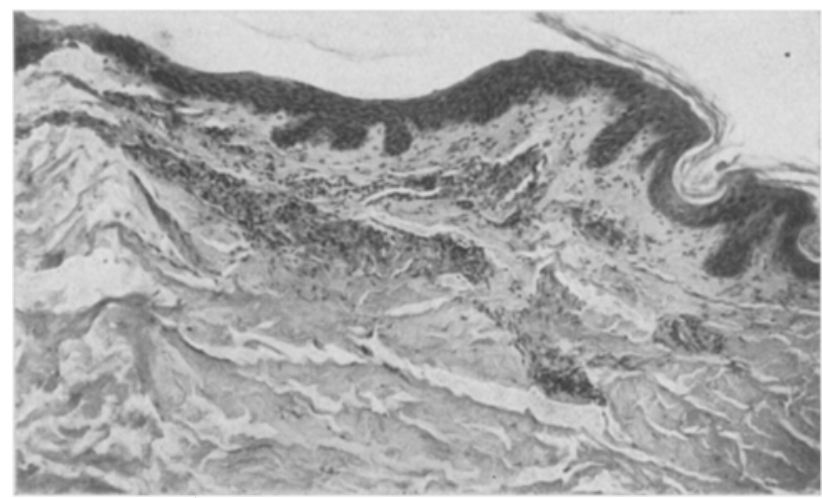

Abb. 7. Starker Zellreichtum der Cutis in einem makroskopisch als Petechie imponierenden Herd bei Pneumokokkämie.

Stellen Extravasate überhaupt nicht vorlagen. Ähnliches habe ich bei einem der von mir beobachteten Fälle von petechialem Exanthem bei Genickstarre (l. c.) berichtet. Dort war Stase im Bereich eines, auf eine kurze Strecke und nicht in ganzem Umfang, die Zeichen der Nekrose aufweisenden Arterienastes, und so der Eindruck einer Hämorrhagie entstanden. Hier lag eine erheblich geringere Schädigung der Gefäßwand vor. Aber daß es sich um eine solche handelte, ist durch das Auftreten teils hyaliner, teils aus Leukocyten bestehender Thromben und durch die hier sehr erhebliche Proliferation fixer Gewebszellen erhärtet. Der Nachweis von Krankheitserregern schlug auch hier fehl. Aber ich glaube, daß die im 1. Falle in dieser Beziehung geführte Beweisführung auch hier Geltung haben dürfte, zumal die histologischen Veränderungen, abgesehen von dem Fehlen eines freien Extravasats, hier sonst im wesentlichen übereinstimmen. Die Entstehung der letzteren sehe ich also auch hier als Effekt des in die Haut eingedrungenen, 
aber zur Zeit des 'Todes des Pat. dort nicht mehr auffindbaren Diploc. lanz. an.

Die fortgesetyte systematische Kontrolle der Haut bej akuten infektiösen Allgemeinerkrankungen hat, wie ich durch das vorstehend mitgeteilte Beobachtungsmaterial bewiesen zu haben glaube, eine weitere Reihe bisher unbekannter Tatsachen zutage gefördert. Bei den beiden Grundleiden, um die es sich hier handelte, der eitrigen Meningitis und der Lungenentzünd ung, bot die Haut für die Betrachtung mit dem bloBen Auge scheinbar gleichartige Verände-

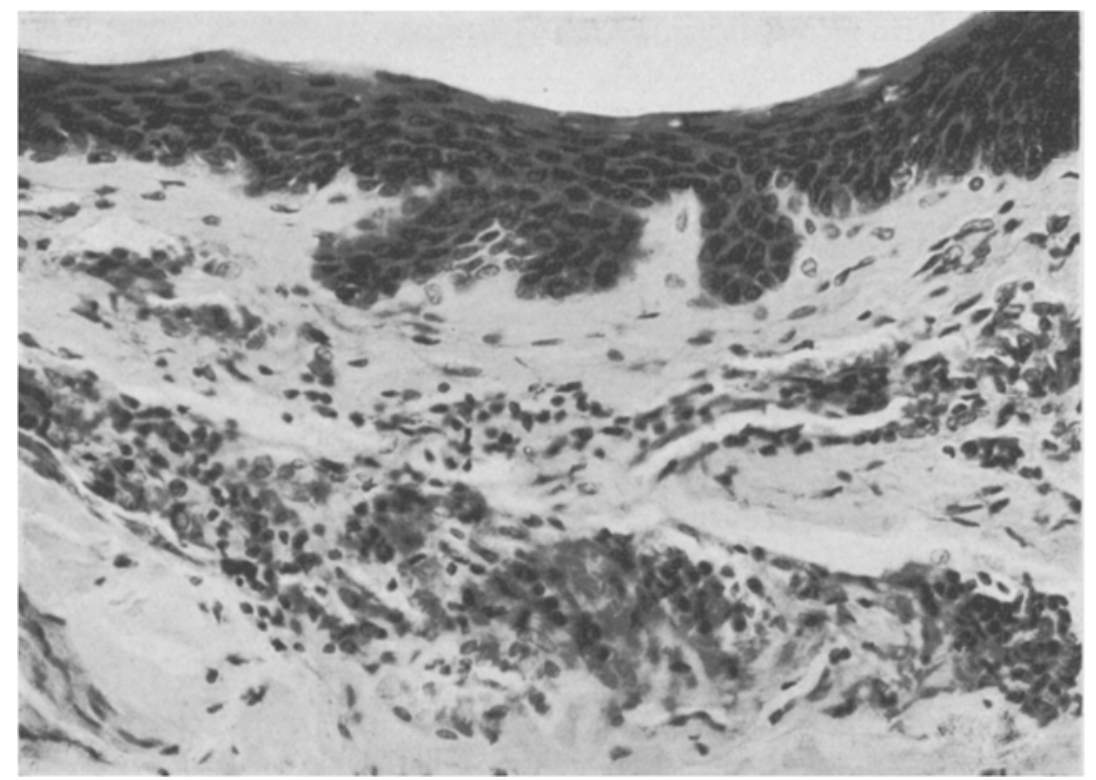

Abb. 8 . Lenkocytenthrombus im miteren Ast der Gabel der Präcapillare aus don als Petechiv. imponierenden Herd in der Hant bei P’nennokokkämie: stärkere Vergrößerung der Abb. 7 .

rungen, nämlich hämorrhagischeZustände. Die mikroskopische Untersuchung hat uns indes darüber belehrt, daß das diese $m$ makroskopischen Aussehen zugrunde liegende anatomische Substrat cin bei den einzelnen fällen ganz verschiedenes war. Die hochgradigsten Veränderungen fanden sich in den von der Meningokokken-Meningitis stammenden Petechien. Hier war es zu schweren, bis zu umschriebener Nekrose des kollagenen Gewebes gesteigerten, von leukocytärer Exsudation begleiteten entzündlichen. Vorgängen gekommen, die auf das Eindringen der in und zwischen den Exsudatzellen gelegenen Krankheitserreger zurückgeführt werden konnten. 
Ein wesentlich anderes Bild boten die Petechien bei der Friedländermeningitis. Zwar handelte es sich auch hier keineswegs um reine Hämorrhagien, es ließen sich vielmehr ausgesprochen entzündliche Veränderungen in Form alterativer, einzelne Gefäßchen betreffender und proliferativer Vorgänge an den fixen Gewebselementen der Subcutis feststellen, über deren Abhängigkeit von dem Eindringen des der Meningitis zugrunde liegenden Krankheitserregers in das Unterhautgewebe nicht der geringste Zweifel obwalten konnte.

Im Gegensatz dazu waren die Veränderungen an den Petechien der beiden Fälle von Pneumonieim ganzen geringgradig. Bemerkenswert erscheint vor allem, daß im 2. Fall die, makroskopisch sowohl am Krankenbett als am Sektionstisch, als Extravasate gedeuteten Herde nichts von Blutaustritt erkennen ließen, daß man es vielmehr mit einer auf einen winzigen Bezirk der Cutis beschränkten Erweiter ung und starken Füllung der hier verlaufenden Präcapillaren, mit einer erheblichen Schwellung und Vermehrung der fixen Gewebszellen des Corium, sowie mit dem Auftreten von teils hyalinen, teils aus meist ein kernigen Zellen zusam mengesetzten Thromben in einzelnen Gefäßchen zu tun hatte. Im Gegensatz dazu fand sich im 1. Fall ein, freilich nur wenig dichtes, Extravasat an der Grenze von $P$. papill. u. reticul. cut. in der unmittelbaren Umgebung einer sich gabelig teilenden, einen hyalinen, wandständigen Thrombus aufweisenden Präcapillare. Es können also makroskopisch als Blutaustritte in die Haut oder Subcutis imponierende Flecke ein durchaus differentes Verhalten darbieten, wie die an Hautpetechien bei der Meningokokken- und Friedländermeningitis, bei der Rachendiphtherie, bei der Pneumonie erhobenen Befunde einwandfrei bewiesen haben. In nicht wenigen dieser Fälle handelt es sich nicht um reine Extravasate, sondern um mit Hämorrhagien vergesellschaftete entzündliche Prozesse, die fast regelmäßig deutliche, ihrem Wesen nach freilich nicht nur bei den einzelnen Krankheiten, sondern auch bei Fällen des gleichen Grundleidens keineswegs übereinstimmende Alterationen der Gefäßwand erkennen lassen.

Als irgendwie spezifisch können die hier geschilderten Veränderungen in den Hautpetechien bei der Pneumonie und bei verschiedenen Meningitisformen nicht angesehen werden. Sie gestatten in keinem Fall eine Diagnose a uf das ihnen zugrunde liegende Allgemeinleiden. Ganz im Gegensatz zu den charakteristischen Befunden an den Roseolen beim Fleckfieber, deren histologische Untersuchung die sichere Erkennung eines Falles als Fleckfieber zuläßt, ganz im Gegensatz ferner zu den Roseolen beim Unterleibstyphus durch den Nachweis der Krankheitserreger 
in einer für sic charakteristischen Anordnung in der vorher bebrüteten Haut, ganz im Gegensatz endlich zu den hämorrhagischnekrotischen, als Ekthyma gangränosum bezeichneten Efflorescenzen, bei denen durch die, geradezu pathognomonische Ansiedlung der Krankheitserreger in den Wandungen von Arterien und Venen der ergriffenen Hautbezirke, allein aus dem mikroskopischen Bild, ohne Zuhilfenahme des Kulturverfahrens, die Allge meiner kran kung und die Art des ihr zugrunde liegenden Erregers erkannt werden kann.

In allerjüngster Zeit hat Lüd ke bei Fällen von Allgemeininfektion durch den Mikrokok. tetrag. das Auftreten eines, wie es scheint auch sehr charakteristischen, hämorrhagisch werdenden Exanthems beobachtet (Münch. med. Wochenschr. 1920, Nr. 16), über dessen histologisches Verhalten, spez. über die Beziehungen der Krankheitserreger zu dem affizierten Hautgewebe, Angaben leider fehlen. Es wäre dringend erwünscht, falls sich die Gelegenheit zu ähnlichen Beobachtungen bietet, diese Lücke baldigst auszufüllen. Auch die Lüdkesche Mitteilung zeigt, in wie wechselnder Weise die Haut auf das hämatogene Eindringen der verschiedenen Krankheitserreger bei akuten Allgemeininfektionen reagiert. Ich zweifle nicht daran, daß durch fortgesetzte Untersuchungen auf diesem Gebiet eine Erweiterung unserer Kenntnisse hinsichtlich mancher anderen, den Verlauf der hier erörterten Allgemeinerkrankungen begleitenden, bisher gänzlich unerforschten, ihrem Wesen nach deshalb völlig unklaren, nicht petechialen Hautaffektionen zu erwarten ist. 